\title{
Home Blood Pressure Measurement in the Morning Related to Cancer in Patients with Type 2 Diabetes Mellitus: 10 Years-Term Results of a Longi- tudinal Study and Cancer
}

\author{
Kyuzi Kamoi*
}

The Center Diabetes \& Endocrinology Metabolism, Joetsu General Hospital, Joetsu, Niigata 943-8502, Japan

\begin{abstract}
Previous longitudinal studies have demonstrated that blood pressure measurements at home (HBP) in the wakening-up display stronger predictive power for death, and vascular complications in patients with type 2 diabetes mellitus (T2DM) than clinic blood pressure measurements (CBP). The leading cause of death was cancer. Patients with T2DM have associated with cancer, and high CBP is a risk factor for cancer. Therefore, this study investigated whether HBP or CBP is related to cancer event in patients with T2DM for 10 years. At baseline, 400 Japanese patients with T2DM were classified as hypertensive (HT) or normotensive (NT) based on HBP and CBP. Mean ( \pm SD) duration was $95 \pm 35$ months. Primary and secondary endpoints were death and cancer, respectively. Differences in outcome between HT and NT were analyzed using survival curves from Kaplan-Meier analysis and log-rank testing. Associated risk factors were assessed using Cox proportional hazards. On basis of HBP, death and event of cancer were significantly higher in HT than in NT. The leading cause of death was cancer. On basis of CBP, there was no significant difference in the incidence of death and event of cancer between patients with HT and NT at baseline. Associated risk factor for cancer was T2DM. Home morning HT may be reflected more keenly state of cancer than clinic HT, which may be superior to clinic NT. When we meet with such patients, it is important that cancer may be one of many causes for morning HT in Japanese patients with T2DM.
\end{abstract}

Keywords: Cancer, clinic blood pressure, morning home blood pressure, risk factor, type 2 diabetes mellitus.

\section{INTRODUCTION}

Previous cross-sectional studies [1,2], and 6-year [3] and 10-year [4] longitudinal studies have demonstrated that home blood pressure (HBP) measurements upon awakening have better predictive power for death and micro- and macrovascular complications in patients with type 1 and 2 diabetes mellitus (T2DM) than casual/clinic BP (CBP) measurements.

The leading cause of death in longitudinal studies was cancer. From old time, it was known that patients with T2DM have associated with cancer $[5,6]$, although the mechanism was not clear [7]. Further, high CBP (clinic hypertension: $\mathrm{CH}$ ) is a risk factor for cancer [8], although the mechanism was also not clear $[9,10]$.

Therefore, a present prospective and longitudinal study examined whether HBP or CBP measurements provided stronger reflection to outcome of cancer by comparing cumulative event between patients with T2DM associated with hypertension (HT) or normotension (NT) over 10 years.

\section{RESEARCH DESIGN AND METHODS}

\section{Subjects}

Subjects comprised 400 Japanese patients with T2DM enrolled between 1999 and 2005 [3]. After a detailed

\footnotetext{
*Address correspondence to this author at the Joetsu General Hospital, Joetsu, Niigata, 943-0502, Japan; Tel: +81-0258-36-3986; Fax: +81-0258-36-3986; E-mail: kkam-int@echigo.ne.jp
}

baseline examination, subjects were followed up for allcause mortality and morbidity, and event of cancer. All participants visited our clinic regularly and were followed until March 31, 2013. T2DM was diagnosed according to the World Health Organization criteria [11], which nowadays require glycated hemoglobin (HbAlc) level as National Glycohemoglobin Standardization Program according to the Japan Diabetes Society [12] is greater than $6.5 \%$.

Baseline characteristics of participants have been described previously [3]. At beginning of the study, 329 patients $(82 \%)$ were receiving treatment with oral hypoglycemic drugs and/or insulin regimens for hyperglycemia, 196 patients $(49 \%)$ were receiving treatment with various antihypertensive drugs for HT, and no patient was receiving treatment for anemia $[3,4]$.

All patients were fully informed about purposes and procedures for the study and provided oral consent at enrolment. This study was a registered clinical trial (Clinical Trial reg. no. NCT00760110).

\section{Baseline and Follow-up Assessment}

Participants were examined using the methods reported previously [3]. All chemical laboratory data were obtained at each clinic visit in a non-fasting state. A single specimen at each visit was used to assess urinary albumin levels based on 2009 guidelines of the American Diabetes Association [13]. Microalbuminuria and clinical albuminuria were defined as urinary albumin excretion rate $\geq 30 \mathrm{mg} / \mathrm{g}$ creatinine and $\geq 300$ $\mathrm{mg} / \mathrm{g}$ creatinine, respectively [13]. 
CBP was measured once in each clinic visit [3]. A1though HBP was measured every day in the morning within 10 minutes after awakening in the sitting position, HBP value assessed for this study used the value measured once in the same morning at each clinic visit $[3,4]$.

$\mathrm{CH}$ and home morning hypertension $(\mathrm{HMH})$ were defined as systolic blood pressure (SBP) $\geq 130 \mathrm{mmHg}$ and/or diastolic blood pressure (DBP) $\geq 85 \mathrm{mmHg}$, respectively, whereas clinic normotension $(\mathrm{CN})$ and home morning normotension (HMN) were defined as SBP $<130 \mathrm{mmHg}$ and DBP $<85 \mathrm{mmHg}$, respectively. The reason of same threshold used for both clinic and morning values was based on criteria of the 1999 World Health Organization-International Society of Hypertension guidelines [14] because this study started in 1999.

At baseline, HT or NT was determined on basis of HBP and CBP measurements. Based on HBP, subjects were divided into patients with $\mathrm{HMH}$ and $\mathrm{HMN}$, and antihypertensive drug use at baseline and end-point was determined in each group $[3,15]$. Sustained HMH and sustained HMN were defined when values met the definitions of blood pressure from baseline through end-point [3]. In addition, based on $\mathrm{CBP}$, subjects were divided into $\mathrm{CH}$ and $\mathrm{CN}$ patients. These patients were followed using same methods used for patients with HMH or HMN [3, 4].

Microvascular complications included nephropathy, neuropathy and retinopathy. Macrovascular complications were consisted of coronary heart disease, cerebrovascular disease, and peripheral artery obstruction $[3,4]$. Event for cancer was classified by its anatomic origin and diagnosed by symptoms and laboratory findings [5].

For ethical reasons, patients were treated with various anti-hypertensive, anti-diabetic, anti-dyslipidemic, antihypercoagulative, and anti-anemic agents during course of the study by their own doctors $[3,4]$.

\section{Study End-points and Outcome Measures}

Outcome results considered only the first event in each subject $[3,15]$.

The primary end-point was death from any cause [3, 4]. The death of patients was confirmed by author self and then patients had been checked values of HBP at wakening-up and CBP one day before death based on the medical record.

The secondary end-point was cancer event. The event of caner was defined as diagnostic cancer using the medical records when they had medical examination because of their bad way. Therefore, it was not defined as mortality. The values of HBP and CBP measured were examined on the day of diagnosis based on the medical record. Information of cancer in some patients informed author from their acquaintances.

\section{Risk Factor Assessment for Outcomes}

Risk factors included aging, gender and obesity (BMI) in baseline and end-point related to cancer event as reported previously were determined [9]. Laboratory variables as HbA1c, triglyceride, total cholesterol, high density lipoprotein, low density lipoprotein, and creatinine in serum, and urinary albumin excretion rate were also determined as risk factors. Chronic complications, and therapies of before (baseline therapy) and after conducting a follow-up survey (additional therapy) were determined as additional risk factors. The 6-month interval minimizes bias owing to the fall or the rise in HBP or CBP measurement [15].

\section{STATISTICAL METHODS}

\section{Baseline}

All results are presented as means \pm SD. Mean values were compared using paired or unpaired student $t$ test. To compare the prevalence of various complications or medical treatments in patients with and without HT on basis of HBP or CBP, Fisher's exact test with two-tailed P values was used $[3,4]$.

\section{End-points and Outcome Measures}

Differences in outcomes for each end-point of death and event of cancer between patients with HT and NT on basis of HBP or CBP measured at baseline and end-point [3, 4] were assessed using Kaplan-Meier survival curves and then compared by hazard ratio using log-rank test, in which hazard ratio and $95 \%$ confidence intervals were calculated $[3,4]$.

\section{Risk Factor Assessment for Outcomes}

Risk factors excluded blood pressure, which determined to be related to outcome were assessed by Cox proportional hazard analysis [3, 4].

Analysis was performed using Prism version 6.03 software (GraphPad Software, CA, USA) and R version 3.0.2 (The R Foundation for Statistical Computing, Vienna, Austria). Two-tailed values of $\mathrm{P}<0.05$ were considered statistically significant.

\section{RESULTS}

\section{Baseline Characteristics of Patients}

Baseline characteristics of patients classified as HT or NT on basis of HBP and CBP were described previously [3].

\section{End-points and Outcome Measures}

Based on HBP, 286 patients (71.5\%) with HMH at baseline were deceased to be 122 patients $(31 \%)$ with $\mathrm{HMH}$ at end-point (Tables 1 and 2). On the other hand, 114 patients $(28.5 \%)$ with HMN at baseline were increased to be 278 patients (70\%) with $\mathrm{HMN}$ at end-point (Tables 1 and 2). Among them, 172 patients (43\%) with $\mathrm{HMH}$ at baseline and 68 patients $(17 \%)$ with $\mathrm{HMH}$ at end-point were received anti-hypertensive drugs, respectively, whereas 22 patients (5.5\%) with HMN at baseline and 126 patients $(31.5 \%)$ with HMN at end-point were received anti-hypertensive drugs, respectively. Sustained $\mathrm{HMH}$ was seen in 100 patients $(27 \%)$, whereas sustained HMN was seen in 92 patients (23\%). Namely, 186 patients $(46.5 \%)$ changed from $\mathrm{HMH}$ to $\mathrm{HMN}$, while 22 patients $(5.5 \%)$ changed from $\mathrm{HMN}$ to $\mathrm{HMH}$.

Based on CBP, 283 patients $(71 \%)$ with $\mathrm{CH}$ at baseline were decreased to be 117 patients $(29.5 \%)$ with $\mathrm{CH}$ at endpoint (Table 1). On the other hand, 172 patients (43\%) with 
Table 1. Primary and secondary outcomes based on home blood pressure (HBP) and clinic blood pressure (CBP) measured at baseline.

\begin{tabular}{|c|c|c|c|c|c|c|c|c|}
\hline Outcome & \multicolumn{4}{|c|}{ Patient status on HBP at baseline $(n=400)$} & \multicolumn{4}{|c|}{ Patient status on CBP at baseline $(n=400)$} \\
\hline & $(n=286)$ & $(n=114)$ & $(95 \% \mathrm{Cl})$ & & $(n=283)$ & $(n=117)$ & $(95 \% \mathrm{Cl})$ & \\
\hline $\begin{array}{l}\text { Primary outcome } \\
\text { Death }\end{array}$ & 66 & 14 & $1.7(1.1-2.5)$ & $0.0001^{*}$ & 50 & 30 & $0.7(0.4-2.2)$ & 0.2172 \\
\hline $\begin{array}{c}\text { Secondary outcome } \\
\text { Cancer }\end{array}$ & 53 & 12 & $1.9(1.0-3.0)$ & $0.0251^{*}$ & 49 & 16 & $1.2(0.7-2.0)$ & 0.5582 \\
\hline
\end{tabular}

Characteristics of patients at baseline on basis of HBP and CBP measurement are shown in a report described previously. The 400 patients in each group were classified as having hypertension or normotension according to values of blood pressure measured in the home or in the clinic at start of this study, respectively. Differences in primary and secondary outcomes for new or worsened events of endpoint between hypertensive patients and normotensive patients measured at baseline in each group were assessed using survival curves from the Kaplan-Meier method, and comparisons were analyzed using hazard ratios by the log-rank test. *Significant difference between hypertensive and normotensive patients. CI, confidence interval.

Table 2. Primary and secondary outcomes based on home blood pressure (HBP) and clinic blood pressure (CBP) measured at endpoint.

\begin{tabular}{|c|c|c|c|c|c|c|c|c|}
\hline Outcome & \multicolumn{4}{|c|}{ Patient status on HBP at endpoint $(n=400)$} & \multicolumn{4}{|c|}{ Patient status on CBP at endpoint $(n=400)$} \\
\hline & $(n=122)$ & $(n=278)$ & $(95 \% \mathrm{Cl})$ & & $(n=172)$ & $(n=228)$ & $(95 \% \mathrm{Cl})$ & \\
\hline $\begin{array}{l}\text { Primary outcome } \\
\text { Death }\end{array}$ & 41 & 39 & $2.7(1.9-3.9)$ & $0.0001 *$ & 47 & 33 & $1.9(1.3-3.1)$ & $0.0024 *$ \\
\hline $\begin{array}{c}\text { Secondary outcome } \\
\text { Cancer }\end{array}$ & 27 & 38 & $2.1(1.3-4.1)$ & $0.0014 *$ & 35 & 30 & $1.8(1.1-2.9)$ & $0.0110^{*}$ \\
\hline
\end{tabular}

The 400 patients in each group were classified as having hypertension or normotension according to values of blood pressure measured in the HBP or in CBP at endpoint of this study, respectively. Differences in primary and secondary outcomes for new or worsened event of endpoint between patients with hypertension and normotension measured at endpoint in each group were assessed using survival curves from the Kaplan-Meier method, and comparisons were analyzed using hazard ratios by the log-rank test. * Significant difference between hypertensive and normotensive patients. CI, confidence interval.

$\mathrm{CN}$ at baseline were increased to be 228 patients (57\%) with $\mathrm{CN}$ at end-point (Table 2). Among them, 172 patients (43\%) with $\mathrm{CH}$ at baseline and 68 patients $(17 \%)$ with $\mathrm{CH}$ at endpoint were received anti-hypertensive drugs, respectively, whereas 22 patients $(5.5 \%)$ with $\mathrm{CN}$ at baseline and 126 patients $(31.5 \%)$ with $\mathrm{CN}$ at end-point were received antihypertensive drugs, respectively. Sustained $\mathrm{CH}$ was seen in 148 patients (37\%), whereas sustained $\mathrm{CN}$ was seen in 84 patients (21\%). Namely, 134 patients $(33.5 \%)$ changed from $\mathrm{CH}$ to $\mathrm{CN}$, while 34 patients $(8.5 \%)$ changed from $\mathrm{CN}$ to $\mathrm{CH}$. The remaining subjects did not change.

In terms of primary outcome, the detailed status was described previously [4]. Briefly, 80 cumulative deaths $(20 \%)$ were observed over 10 years. Cause of death in 36, 20, 13, and 11 patients was cancer, CVD, CHD, and unknown, respectively. Among them, 32, 15, 10, and 9 patients $(\mathrm{n}=66$; $16.5 \%$ ) with $\mathrm{HMH}$ at baseline, and 27, 6, 5 and 3 patients $(\mathrm{n}=41)(10 \%)$ with HMH at end-point were due to the same diseases, respectively.

The hazard ratio of primary outcome was significantly higher in HMH group than HMN group at baseline and endpoint (Tables 1 and 2). There were no significant differences in the events between $\mathrm{CH}$ and $\mathrm{CN}$ groups at baseline, but significant difference appeared between these groups at endpoint (Tables $\mathbf{1}$ and 2). However, the value of significant difference was significantly $(\mathrm{P}<0.001)$ lower in $\mathrm{CBP}$ than in HBP (Table 2).
In terms of secondary outcome, mean survey duration until end-point was $92 \pm 78$ months (range 5-120 months). At baseline, two patients had cancer $(0.5 \%)$; one patient had colon cancer and other patient had brain tumor. However, over 10 years, cancer event was observed in 65 patients $(16 \%)$.

The hazard ratio of secondary outcome was significantly higher in HMH group than in HMN group at baseline (Table 1) and end-point (Table 2), although there was no significant difference in the event between $\mathrm{CH}$ and $\mathrm{CN}$ groups at baseline (Table 1). However, significant difference appeared between these groups at end-point (Tables $\mathbf{1}$ and 2). The value of significant difference was also significantly ( $P$ $<0.001$ ) lower in CBP than in HBP (Table 2). Among them, all patients $(100 \%)$ with $\mathrm{HMH}$ at baseline, 17 patients $(63 \%)$ with $\mathrm{HMH}$ at end-point, 27 patients $(55 \%)$ with $\mathrm{CH}$ at baseline and 28 patients $(80 \%)$ with $\mathrm{CH}$ at end-point were received anti-hypertensive drugs, respectively.

\section{Risk Factor Assessment for Outcomes}

In terms of cancer's event, there were no confounding risk factors shown in baseline for patients with HMH measured at baseline (Table 3). Additional therapy for HT and non-insulin therapy for hyperglycemia in end-point were also risk factors in patients with HMH measured at endpoint, although the hazard ratio in additional therapy for HT was less than 1.0 (Table 4). There were no significantly risk factors in other variables. 
Table 3. Risk factors for cancer event in patients with home morning hypertension (HMH) on basis of home blood pressure (HBP) measured at baseline.

\begin{tabular}{|c|c|c|}
\hline Variable & $\begin{array}{c}\text { Hazard } \\
\text { ratio }\end{array}$ & P value \\
\hline Age (years) & 1.01 & 0.711 \\
\hline Gender (Female/Male) & 0.52 & 0.079 \\
\hline Duration (years) & 0.97 & 0.109 \\
\hline $\operatorname{BMI}\left(\mathrm{kg} / \mathrm{m}^{2}\right)$ & 0.92 & 0.153 \\
\hline \multicolumn{3}{|l|}{ Laboratory variables } \\
\hline $\mathrm{HbA} 1 \mathrm{c}(\%)$ & 1.02 & 0.921 \\
\hline Triglycerides (mg/dl) & 1.00 & 0.364 \\
\hline Total cholesterol (mg/dl) & 1.01 & 0.195 \\
\hline LDL-cholesterol (mg/dl) & 1.00 & 0.150 \\
\hline HDL-cholesterol (mg/dl) & 1.00 & 0.927 \\
\hline Serum creatinine (mg/dl) & 1.55 & 0.139 \\
\hline Urinary albumin excretion ( $\mathrm{mg} / \mathrm{g}$ creatinine) & 1.00 & 0.269 \\
\hline \multicolumn{3}{|l|}{ Medical events } \\
\hline Microvascular complications & 1.01 & 0.918 \\
\hline Macrovascular complications & 0.79 & 0.463 \\
\hline \multicolumn{3}{|l|}{ Medical treatments } \\
\hline \multicolumn{3}{|l|}{ Therapy for hypertension } \\
\hline Baseline & 0.87 & 0.465 \\
\hline Additional & 0.85 & 0.237 \\
\hline \multicolumn{3}{|l|}{ Therapy for diabetes mellitus } \\
\hline \multicolumn{3}{|l|}{ Baseline } \\
\hline Non-insulin agents & 0.84 & 0.423 \\
\hline Insulin Therapy & 1.15 & 0.725 \\
\hline \multicolumn{3}{|l|}{ Additional } \\
\hline Non-insulin agents & 0.95 & 0.783 \\
\hline Insulin Therapy & 0.86 & 0.757 \\
\hline \multicolumn{3}{|l|}{ Therapy for dyslipidemia } \\
\hline Baseline & 0.67 & 0.660 \\
\hline Additional & 2.46 & 0.424 \\
\hline \multicolumn{3}{|l|}{ Therapy for hypercoagulation and others } \\
\hline Baseline & 2.75 & 0.424 \\
\hline Additional & 0.16 & 0.135 \\
\hline
\end{tabular}

Cancer event was determined in patients with HMH on basis of HBP measured at baseline, which was determined by the log-rank test. Confounding factors at baseline were reported previously and were added as laboratory variables, chronic complications and therapies of before (baseline therapy) and after (additional therapy) conducting a follow-up survey, as additional risk factors. Blood pressures as a confounding factor were excluded because that they had significant in shown table 1. Associated risk factors among the confounding factors were assessed using hazard ratio by Cox proportional hazards modeling. Two-tailed value of $\mathrm{P}<0.05$ was considered statistically significant as a confounding factor.
Table 4. Risk factors for cancer event in patients with home morning hypertension (HBP) on basis of home blood pressure (HBP) measured at end-point.

\begin{tabular}{|c|c|c|}
\hline Variable & $\begin{array}{c}\text { Hazard } \\
\text { ratio }\end{array}$ & P value \\
\hline Age (years) & 1.06 & 0.210 \\
\hline Gender (Female/Male) & 0.42 & 0.185 \\
\hline Duration (years) & 0.98 & 0.620 \\
\hline $\operatorname{BMI}\left(\mathrm{kg} / \mathrm{m}^{2}\right)$ & 0.99 & 0.908 \\
\hline \multicolumn{3}{|l|}{ Laboratory variables } \\
\hline $\mathrm{HbA1c}(\%)$ & 0.65 & 0.194 \\
\hline Triglycerides (mg/dl) & 1.00 & 0.437 \\
\hline Total cholesterol (mg/dl) & 1.02 & 0.254 \\
\hline LDL-cholesterol (mg/dl) & 0.98 & 0.166 \\
\hline HDL-cholesterol (mg/dl) & 0.99 & 0.729 \\
\hline Serum creatinine $(\mathrm{mg} / \mathrm{dl})$ & 2.01 & 0.210 \\
\hline Urinary albumin excretion (mg/g creatinine) & 1.00 & 0.437 \\
\hline \multicolumn{3}{|l|}{ Medical events } \\
\hline Microvascular complications & 1.11 & 0.641 \\
\hline Macrovascular complications & 0.99 & 0.989 \\
\hline \multicolumn{3}{|l|}{ Medical treatments } \\
\hline \multicolumn{3}{|l|}{ Therapy for hypertension } \\
\hline Baseline & 1.04 & 0.892 \\
\hline Additional & 0.46 & 0.005 \\
\hline \multicolumn{3}{|l|}{ Therapy for diabetes mellitus } \\
\hline \multicolumn{3}{|l|}{ Baseline } \\
\hline Non-insulin agents & 3.27 & 0.011 \\
\hline Insulin Therapy & 3.82 & 0.097 \\
\hline \multicolumn{3}{|l|}{ Additional } \\
\hline Non-insulin agents & 1.15 & 0.645 \\
\hline Insulin Therapy & 0.24 & 0.150 \\
\hline \multicolumn{3}{|l|}{ Therapy for dyslipidemia } \\
\hline Baseline & 0.07 & 0.160 \\
\hline Additional & 5.41 & 0.304 \\
\hline \multicolumn{3}{|l|}{ Therapy for hypercoagulation and others } \\
\hline Baseline & 2.26 & 0.992 \\
\hline Additional & 2.27 & 0.992 \\
\hline
\end{tabular}

Cancer event was determined in patients with MH on basis of HBP at end-point, which was determined by the log-rank test. Confounding factors at end-point were same in the baseline characteristics of participants as report previously and were added as laboratory variables, chronic complications and therapies of before (baseline therapy) and after (additional therapy) conducting a follow-up survey, as additional risk factors. Blood pressures as a confounding factor were excluded because that they had significant in shown table 2. Associated risk factors among the confounding factors were assessed using hazard ratio by Cox proportional hazards modeling. Two-tailed value of $\mathrm{P}<0.05$ was considered statistically significant as a confounding factor. 


\section{CONCLUSION}

Recent guidelines have recommended that threshold of blood pressure for HBP should be lower by $5-10 \mathrm{mmHg}$ than that for CBP [16-18]. In this study, the same thresholds for HBP and CBP in groups were used based on guidelines in place when this study began $[3,4]$. Nevertheless, the cumulative event of death was about twice as high in patients with HMH compared to patients with HMN [4]. The leading cause of death was due to cancer as previous reports [3, 4].

In terms of cancer's event, this study indicates that although in baseline incidence of cancer was small, the incidence was increased over 10 years, and elevated blood pressure may be related to cancer, particularly HMH. Further, the result showed that confounding factor is diabetes mellitus, as confounding factor is also additional therapy for HT, but the hazard ratio was less than 1.0. Although many mechanisms of a relation with hypertension and cancer were proposed $[9,10]$, the detailed mechanism is unknown. If cancer may give an internal stress to such patients, the stress may induce elevation of blood pressure. Thus, the elevated blood pressure may reflect sharper in $\mathrm{HMH}$ than $\mathrm{CH}$.

All findings indicated that HMH may be keenly reflected the state of cancer event in patients with T2DM than $\mathrm{CH}$. When we meet with such patients, it is important that cancer may be one of many causes for HMH in Japanese patients with T2DM.

\section{LIMITATIONS OF THIS STUDY}

First, the numbers of patients participated and the events occurring over 10 years were heterogeneous and small. Therefore, we were unable to survey outcomes and compare differences among groups of patients with $\mathrm{HMH}, \mathrm{HMN}, \mathrm{CH}$ and $\mathrm{CN}$ measured at baseline as a cohort study, which would have provided clinically valuable information.

Second, there was no evening or 24-hour blood pressure measurement. Instead, we classified patients into HT or NT groups based on HBP and CBP, and compared differences in cumulative events between these groups. These patients' classifications obviously overlapped.

Third, for ethical reasons, most patients received treatment with anti-hypertensive agents and other medications during follow-up $[3,4]$. Therefore, we were unable to examine outcomes without changing treatments from baseline over 10 years and determine whether these drugs influenced outcomes of events. At baseline, $49 \%$ of subjects were receiving antihypertensive treatment, whereas at end-point, $65 \%$ of subjects were receiving antihypertensive treatment. Conventionally, antihypertensive drugs are prescribed in the day time on basis of CBP, but currently, we prescribe antihypertensive drugs before going to bed on basis of HBP [3, 4]. Therefore, it can be argued that it is not appropriate to classify patients who had been taking antihypertensive drugs into groups with HT and NT, as this may introduce a bias in comparisons between CBP and HBP measurements.

Fourth, the prognostic values of CBP and HBP should be assessed as not only categorical data, but also continuous data. The analysis using continuous variables may give more significant meaning, but in this study, blood pressure as a continuous variable showed high fluctuations and the numbers of participants was small. Accordingly, this type of analysis was not done in this study.

In conclusion, HMH may be reflected more keenly state of cancer event than $\mathrm{CH}$, which is superior to $\mathrm{CH}$. When we meet with such patient, it is important that cancer may be one of many causes for HMH in Japanese patients with T2DM.

\section{CONFLICT OF INTEREST}

The authors confirm that this article content has no conflict of interest.

\section{ACKNOWLEDGEMENTS}

Declared none.

ABBREVIATIONS
$\mathrm{CBP}=$ Clinic blood pressure
$\mathrm{CH}=$ Clinic hypertension
$\mathrm{CN}=$ Clinic normotension
$\mathrm{DBP}=$ Diastolic blood pressure
$\mathrm{HbA1c}=$ Glycated hemoglobin A1c
$\mathrm{HBP}=$ Home blood pressure
$\mathrm{HMH}=$ Home morning hypertension
$\mathrm{HMN}=$ Home morning normotension
$\mathrm{SBP}=$ Systolic blood pressure.
$\mathrm{T} 2 \mathrm{DM}=$ Type 2 diabetes mellitus

\section{REFERENCES}

[1] Kamoi K, Miyakoshi M, Soda S, Kaneko S, Nakagawa O. Usefulness of home blood pressure measurement in the morning in type 2 diabetic patients. Diabetes Care 2002; 25: 2218-23.

[2] Kamoi K, Imamura S, Kobayashi T. Usefulness of home blood pressure measurement in the morning in type 1 diabetic patients. Diabetes Care 2003; 26: 2218-23.

[3] Kamoi K, Ito T, Miyakoshi M, Minagawa S, Kimura K. Usefulness of home blood pressure measurement in the morning in patients with type 2 diabetes: long-term results of a prospective longitudinal study. Clin Exp Hypertens 2010; 32: 184-92.

[4] Kamoi K. Usefulness of morning home blood pressure measurements in patients with type 2 diabetes mellitus: results of a 10-year, prospective, longitudinal study. Clin Exp Hypertens 2014; Apr 30. [Epub ahead of print].

[5] Giovannucci E, Harlan DM, Archer MC, et al. Diabetes and cancer: a consensus report. Diabetes Care 2010; 33: 1674-85.

[6] Yeh HC, Platz EA, Wang NY, Visvanathan K, Helzlsouer KJ, Brancati FL. A prospective study of the associations between treated diabetes and cancer outcomes. Diabetes Care 2012; 35: 113-8.

[7] Zelenko Z, Gallagher EJ. Diabetes and Cancer. Endocrinol Metab Clin North Am 2014; 43: 167-85.

[8] Fraeman HK, Nordstrom LB, Luo W, Sarah HL, Shantakumar S. Incidence of new-onset hypertension in cancer patients: a retrospective cohort study. Int J Hypertens 2013; Article ID 379252; 10.

[9] Hamet P. Cancer and hypertension. An unresolved issue. Hypertension 1996; 28: 321-4.

[10] Mouhayar E, Salahudeen A. Hypertension in cancer patients. Tex Heart Inst J 2011; 38: 263-5.

[11] World Health Organization. Definition, diagnosis and classification of diabetes and its complications. Part 1: diagnosis and classifica- 
tion of diabetes mellitus. Geneva: Department of Noncommunicable Disease Surveillance 1999.

[12] Seino Y, Nanjo K, Tajima N, et al. Report of the committee on the classification and diagnostic criteria of diabetes mellitus. Diabetol Internat 2010; 1: 2-20.

[13] American Diabetes Association. Clinical practice recommendations 2009: Standards of medical care-2009. Diabetes Care (Suppl 1) 2009; 32: S13-S61.

[14] 1999 World Health Organization-International Society of Hypertension Guidelines for the management of hypertension. Guidelines subcommittee. J Hypertens 1999; 17(2): 151-83.

[15] Noguchi Y, Asayama K, Staessen JA, et al. Predictive power of home blood pressure and clinic blood pressure in hypertensive patients with impaired glucose metabolism and diabetes. J Hypertens 2013; 31: 1593-602.

[16] Mancia G, De Backer G, Dominiczak A, et al. Management of Arterial Hypertension of the European Society of Hypertension;
European Society of Cardiology. 2007 Guidelines for the management of arterial hypertension: The task force for the management of arterial hypertension of the European society of hypertension (ESH) and of the European society of cardiology (ESC). J Hypertens 2007; 25: 1105-87.

[17] Pickering TG, Miller NH, Ogedegbe G, Krakoff LR, Artinian NT, Goff D. American Heart Association; American Society of Hypertension; Preventive Cardiovascular Nurses Association. Call to action on use and reimbursement for home blood pressure monitoring: a joint scientific statement from the American Heart Association, American Society of Hypertension, and Preventive Cardiovascular Nurses Association. Hypertension 2008; 52: 10-29.

[18] Ogihara T, Kikuchi K, Matsuoka H, et al. The Japanese Society of Hypertension Guidelines for the management of hypertension (JSH 2009). Hypertens Res 2009; 32: 3-107.

(C) Kyuzi Kamoi; Licensee Bentham Open.

This is an open access article licensed under the terms of the Creative Commons Attribution Non-Commercial License (http://creativecommons.org/licenses/by-nc/3.0/) which permits unrestricted, non-commercial use, distribution and reproduction in any medium, provided the work is properly cited. 